\title{
Transient events in LSST survey data
}

\author{
Astronomical Observatory Belgrade \\ Volgina 7, 11060 Belgrade, Serbia \\ email: jaleksic@aob.rs
}

Jovan Aleksić, Veljko Vujčić and Darko Jevremović

\begin{abstract}
Large Synoptic Survey Telescope will make great contributions to many scientific fields. One of the modules will be time-domain astronomy and detection of transient events. In this paper, some considerations about transient events and alerts are presented.
\end{abstract}

Keywords. LSST, transient event, time-domain astronomy, alert

\section{Introduction}

Large Synoptic Survey Telescope (LSST) will be a large, ground-based, optical telescope that will obtain images over half the sky every few nights (Ivezić et al. 2008). It will contain an $8.4 \mathrm{~m}$ primary mirror, 3.2 Gigapixel camera and will operate in six bands $(u, g, r, i, z$ and $y)$. After 10-years survey period, it is expected that total amount of about 100PB of data will be collected. LSST data products will mostly consist of catalogs and images (Data Products Definition Document). Since they will be used for different purposes, there will be three main categories of data products. Level $\mathbf{1}$ products are intended to detect time-domain events. These data are the product of continuous observations (nightly) followed by analysis of difference images. Variable and moving objects are the result of L1 data products. Level $\mathbf{2}$ products are generated as part of a Data Release. These data are the product of yearly observations, followed by analysis of direct images. Catalogs and images are the result of L2 data products. Level 3 products will be generated by users, using LSST software and/or hardware. Various user-created data will be available that not belong to automatically generated L1 and L2 products (Jurić et al. 2016 (DPDD), Connolly et al. 2013).

\section{Transient events}

A transient astronomical event is the event where the image of observed object changes in time, usually in short time period. Roughly, they can be classified as moving objects, where position changes are detected and variable objects, where flux changes are detected. The objects from former group are characterized by astrometric properties and are related to Solar system objects, while the ones from latter group are characterized by photometric properties and are located beyond the solar system.

If we define the decay time for explosive transients as the period during which the brightness decreases in 2 magnitudes after the peak, majority of them have the decay times of 1 day or longer. The ones with shorter decay time are gamma-ray bursts, which are one of the most brightest events in the universe. Therefore, there exists a large part in M-Td space to explore - for fainter transients with shorter decay time. LSST will be able to explore in this part of parameter space (LSST Science Collaboration, 2009). 


\begin{tabular}{ll}
$\begin{array}{l}\text { Variable objects } \\
\text { (Flux changes) }\end{array}$ & $\begin{array}{l}\text { Moving objects } \\
\text { (Position changes) }\end{array}$ \\
\hline Variable stars & Planets \\
Explosive stars & Asteroids \\
Eclipsing binaries & Comets \\
Transits of extrasolar planets & Trans-Neptunian objects \\
Galaxies & \\
AGN & \\
Bursts (optical) &
\end{tabular}

Table 1. Number of expected transient sources per field (Lynne Jones, 2007)

Cosmic Ray Coincidences $\approx 4000 \mathrm{CR}$ per CCD

Solar System Moving Objects Near ecliptic 2500/field $>5 \sigma$ in $15 \mathrm{~s}$

Solar System Moving Object Near ecliptic, $4500 /$ field $>3 \sigma$ in 30 s

Mdwarf flares

Cataclysmic variables

White Dwarf Pulsators

RR Lyrae's and Cepheids

Delta Scuti Stars

$100 /$ field $>5 \sigma$

$0.6 /$ field $>5 \sigma$

$? ?$

$? ?$

$? ?$

Microlensing Events

Supernova Ia

Gamma Ray Bursts

Toward galactic bulge or Magellanic Clouds, 100-200/field $17 /$ field .004 / field

All kinds of variability, combined 2000/field

Sky Background Noise

Sky Background Noise

$\approx 0.0004$ sources/field above $+5.75 \sigma$, circular aperture 3 pix diam $860 \mathrm{pix} /$ field above $+5 \sigma$

Sky Background Noise

Sky Background Noise

Sky Background Noise

$\approx 0.05$ sources/field above $+5 \sigma$, circular aperture 3 pix diam $\approx 4 \mathrm{e} 6 \mathrm{pix} /$ field above $+3 \sigma$

$\approx 1500$ sources/field above $+3 \sigma$, circular aperture 3 pix diam

Note:

The size of the field is 10 square degrees.

\subsection{Storage principle}

Object properties are not stored as functions of time. Instead, characterizing variables are stored in the database. For moving objects, this is the orbit, defined with 6 orbital elements (q,e,i,lan,aop,M), while for variable objects a variability string determines their behavior. It contains type of variability (RR lyrae, Cepheids, microlensing, etc) alongside with parameters that describe them. Thus, applying orbit or variability string on some particular object, their properties can be obtained for any point in time (position or flux, respectively).

\subsection{Event classification challenge}

At the time of this writing (November 2016), no automatic classification of events exists. The community is asked to contribute with their light curves, type of transient and other contextual information obtained from real observations. The data is collected at the GitHub repository (https://github.com/LSSTTVS/ContributedLCVs) and should be provided as tabulated values. Minimal required information is in the form (time, flux, flux-error, filter), but any additional data useful for characterization is desirable. After collecting the light curves, a set of features for transient and variable objects will be generated. Finally, characterization and classification will be performed, which will be supervised machine learning since the types of variable objects are given. For unsupervised ML, several systems for this task are considered and developed and the main algorithm is as follows. Given the set $\operatorname{mag}(\mathrm{t})$, variability parameters can be obtained using time-series analysis algorithms, such as Lomb-Scargle periodogram. In the next step, 


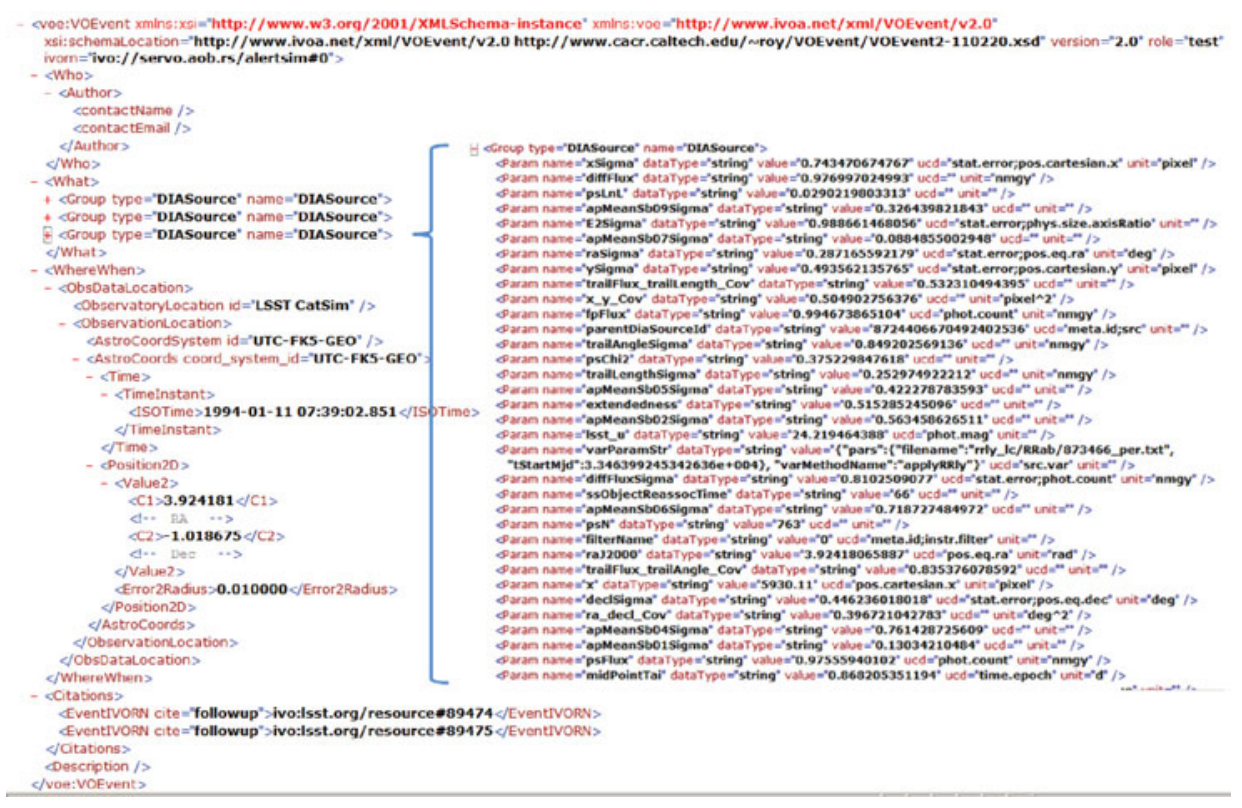

Figure 1. Sample event

classification of physical object types will be performed, based on obtained parameters, using statistical algorithms for classification.

\subsection{Possible changes/improvements}

One single visit is expected to have about 10000 events. Therefore, a lot of identical metadata would be contained within such amount of VOEvents. One idea is to provide "VOEventGroup" or "VOEventContainer" header with metadata about the visit and per-alert data for each particular event. This would significantly reduce the amount of data transmitted.

Another need is the inclusion of "heavyweight" data with the events. Initially, VOEvent is supposed to contain only short, textual data. However, LSST documents proposed cutout images to be included into alerts, so the need for their inclusion emerged.

All these changes are currently being discussed, they are not implemented yet. LSST team will consider to propose IVOA some modifications for VOEvent standard.

An example is shown in the figure 1. The event contains metadata such as observatory (in this case LSST Catalog Simulator), date and time, and sky coordinates. In addition, all previous records of that source are also included. One of the records is expanded, displaying photometric and astrometric properties of the source. Notice the variability parameter string which points to data and method for applying them, allowing the flux to be calculated in any point of time (RR Lyrae in this example). The reader should take care only of the structure and not of particular numbers since they are just randomly generated numbers for populating records during the development phase.

\section{Transient alert}

A Transient Alert is a notification of the detection and characterization of a moving or variable object. Very simple procedure can be described as follows. A visit image is acquired from the telescope. A template image presents what should be seen. Visit image is compared against the template image, and if the difference exists, the alert is raised. 


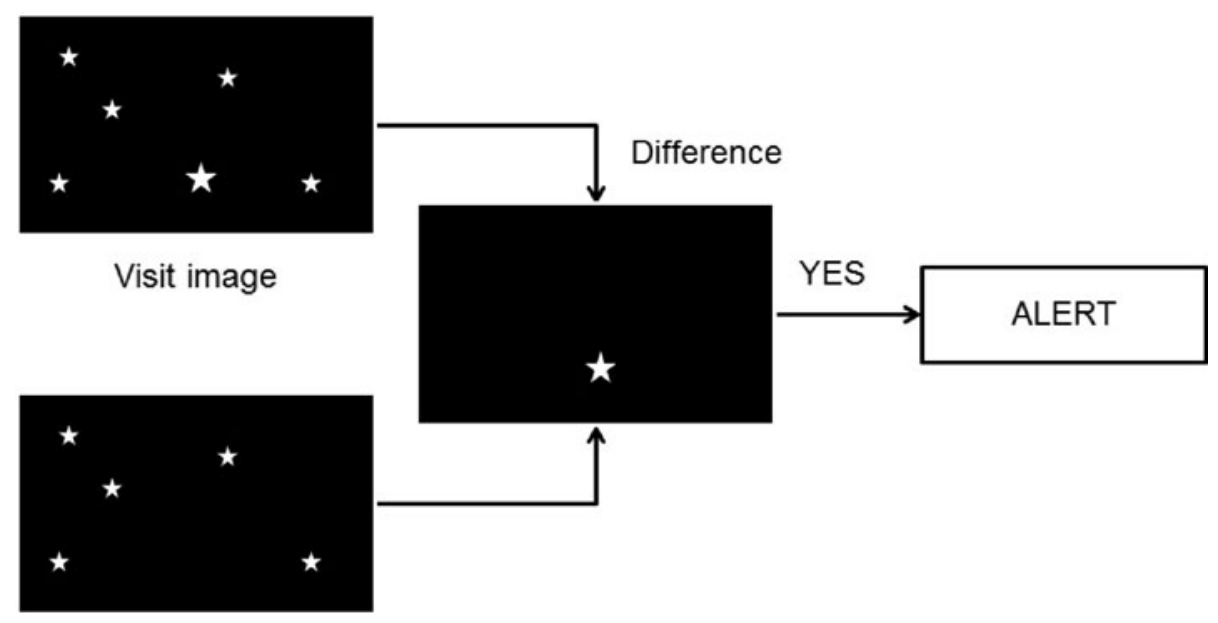

Template image

Figure 2. Basic procedure

This explanation is very simplified. Actually, each of these steps consist several other subprocedures, but the core idea is as described.

A transient alert is a piece of information containing characterization of the object. Each alert contains several data:

- alert ID

- timestamp

- level1 database ID

- Science data

$\circ$ position

$\circ$ flux, size, and shape

- light curves in all bands (up to a year; stretch: all)

o variability characterization (e.g., low-order light-curve moments, probability the object is variable)

- cut-outs centered on the object (template, difference image)

In addition, alerts will have the following properties:

- Alerts will be available world-wide within $1 \mathrm{~min}$ of visit acquisition. This is the result of fast image processing as well as distributing procedures.

- The rate of generating alerts is expected to be quite high, about $10 \mathrm{M}$ per night or about 10k per visit. This assumption is included in design of Alert Simulator process.

- The format should be easy to read and process by variety of systems. Currently considered format is XML, or even better VOEvent (XML record with defined structure, used to describe events in astronomy)

\section{Conclusions}

LSST will play the important role in understanding transient phenomena. Its ability for repetitive observations wide fields of the sky will detect huge amount of interesting events and fast alerting service will help the community for immediate follow-up. We can conclude that LSST will have fundamental contributions to our knowledge of transient and variable universe. 


\section{Acknowledgements}

The authors are grateful for the support provided by Ministry for Education and Science of Republic of Serbia through project III 44002. "Astroinformatics: Application of IT in Astronomy and Close Fields".

\section{References}

Ivezić et al. LSST: from Science Drivers to Reference Design and Anticipated Data Products, arXiv:0805.2366 [astro-ph], 2008.

Jurić, M., et al. LSST Data Products Definition Document, LSE-163, 2016.

Andrew Connolly et al. Requirements for the LSST Simulation Framework, 2013.

LSST Science Collaborations and LSST Project 2009, LSST Science Book, Version 2.0, arXiv:0912.0201,

Becker, A. C. 2008, Transient detection and classification Astron. Nachr., 329, No.3, 280

Borne, K. D. 2008, A machine learning classification broker for the LSST transient database Astron. Nachr., 329, No.3, 255

Mahabal, A. 2008, Automated probabilistic classification of transients and variables Astron. Nachr., 329, No.3, 288

Lynne Jones, 2007

https://github.com/LSSTTVS/ContributedLCVs 